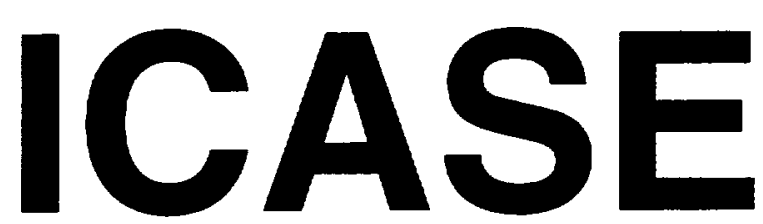

\title{
ENERGY SPECTRUM IN THE DISSIPATION RANGE OF FLUID TURBULENCE
}

\section{D.O. Martinez, S. Chen, G.D. Doolen, R.H. Kraichnan, L.-P. Wang, and Y. Zhou}

NASA Contract No. NASI-19480

May 1996

Institute for Computer Applications in Science and Engineering NASA Langley Research Center Hampton, VA 23681-0001

Operated by Universities Space Research Association



National Aeronautics and Space Administration

Langley Research Center

Hampton, Virginia 23681-0001 



\title{
Energy Spectrum in the Dissipation Range of Fluid Turbulence*
}

\author{
D. O. Martinez ${ }^{1,2,3}, S$. Chen ${ }^{2,1}$, G. D. Doolen ${ }^{1}$, \\ R. H. Kraichnan ${ }^{4}$, L.-P. Wang ${ }^{3}$, and Y. Zhou ${ }^{5}$ \\ ${ }^{1}$ Theoretical Division and Center for Nonlinear Studies \\ Los Alamos National Laboratory, Los Alamos, NM 87545 \\ ${ }^{2}$ IBM Research Division, T.J. Watson Research Center \\ P.O. Box 218, Yorktown Heights, NY 10598 \\ ${ }^{3}$ Department of Mechanical Engineering \\ University of Delaware, Newark, Delaware 19716-3140 \\ ${ }^{4} 369$ Montezuma No. 108, Santa Fe, New Mexico 87501 \\ ${ }^{5}$ ICASE, NASA, Langley Research Center, Hampton, Virginia 23861
}

\begin{abstract}
High resolution, direct numerical simulations of three-dimensional incompressible Navier-Stokes equations are carried out to study the energy spectrum in the dissipation range. An energy spectrum of the form $A\left(k / k_{d}\right)^{\alpha} \exp \left[-\beta k / k_{d}\right]$ is confirmed. The possible values of the parameters $\alpha$ and $\beta$, as well as their dependence on Reynolds numbers and length scales, are investigated, showing good agreement with recent theoretical predictions. A "bottleneck"-type effect is reported at $k / k_{d} \sim 4$, exhibiting a possible transition from near-dissipation to far-dissipation.
\end{abstract}

\footnotetext{
*This work was partially supported by the DOE at Los Alamos and the National Aeronautics and Space Administration under NASA Contract No. NAS1-19480 while one of the authors (Y.Z.) was in residence at the Institute for Computer Applications in Science and Engineering (ICASE), NASA Langley Research Center, Hampton, VA 23681.
} 


\section{Introduction}

The study of the statistical properties of fluid turbulence at small scales is very difficult from both experimental and numerical points of view. In the former, the signal at these scales is exceedingly weak and consequently it is easily contaminated by the background noise that is naturally present in experiments. Resolution of inertial-range and dissipation-range scales at high Reynolds numbers by direct numerical simulations (DNS) requires computational capabilities far beyond those available today.

In the last four decades, several functional forms for the energy spectrum in the dissipation and far-dissipation ranges have been proposed based on heuristic arguments. The energy spectrum in isotropic turbulence can be written as $E(k)=\epsilon^{2 / 3} k^{-5 / 3} F\left(k / k_{d}\right)$, consistent with Kolmogorov's ideas (Kolmogorov 1941). In the expression above, $E(k)=4 \pi k^{2}\left\langle 1 / 2|\hat{u}(k)|^{2}\right\rangle$, where $\hat{u}(k)$ is the Fourier transform of the velocity field; $k_{d}=\epsilon^{1 / 4} \nu^{-3 / 4}$ is the Kolmogorov dissipation wave number, that represents the scale at which the relative importance of the inertial and dissipative terms is comparable; $\epsilon$ is the mean rate of energy dissipation per unit mass, and $\nu$ is the kinematic viscosity. 〈〉 denotes an ensemble average. For scales associated with wavenumbers much smaller than $k_{d}$, the energy spectrum becomes essentially independent of the viscosity $\nu$, and $F\left(k / k_{d}\right)$ is in that limit the Kolmogorov constant $C_{K}$. For wavenumbers $k>k_{d}$, dissipation is important and the function $F$ has a strong dependence on the viscosity through $k_{d}$.

A widely proposed form for $E(k)$ in the dissipation range is $E(k) \sim\left(k / k_{d}\right)^{\alpha} \exp \left(-\beta\left(k / k_{d}\right)^{n}\right)$. There has been considerable debate as to what the values of the parameters $\alpha, \beta$, and $n$ are. Townsend (1951) and Novikov (1961) suggested $n=2$, based on a Taylor expansion of the velocity field at scales much smaller than $\eta \cong 1 / k_{d}$, the dissipation length scale. This same value is preferred by Smith and Reynolds in a more recent work (Smith \& Reynolds 1991) in which they compare the skewness for the longitudinal velocity derivative obtained from experiments, with the values provided by $E(k) \sim\left(k / k_{d}\right)^{\alpha} \exp \left(-\beta\left(k / k_{d}\right)^{n}\right)$ with $n=1,4 / 3$ and 2 , and $\alpha=-5 / 3$. They conclude that $n=2$ is the best fit, and that $n=1$ is a poor model. Sanada (1992) proposed a spectrum with $n=1$, but with a sharp change at a transitional wavenumber. His model fit the spectrum 
from simulations and yielded a skewness of the velocity derivative in agreement with experimental values. Manley (1992) argued that the measurements used by Smith and Reynolds did not extend much beyond the Kolmogorov scale $\eta$, and accordingly he introduced a cutoff wavenumber for the computation of the skewness. With this correction, $n=1$ is a better fit for the experimental data than $n=2$. Kraichnan's direct interaction approximation (DIA) (Kraichnan 1959) predicts $n=1$ for the far-dissipation range; Orszag (1973) argues $n \leq 1$ and Foias et al. conclude $n \geq 1$ (1990) from their analysis. Sreenivasan (1985) found experimentally $n=1$ (for $0.5<k / k_{d}<1.5$ ), and Chen et al. (1993) observed that the same value would best fit the energy spectrum, in a highly resolved DNS at a relatively low Reynolds number. Thus, the value of $n=1$ seems to be established from theories, experiments, and numerical simulations. In the rest of this report, we will concentrate on an energy spectrum of the form

$$
E(k) \sim\left(k / k_{d}\right)^{\alpha} \exp \left(-\beta\left(k / k_{d}\right)\right) .
$$

There are only few studies for determining $\alpha$ and $\beta$. Some phenomenological models (McComb 1990) suggest $\alpha=-5 / 3$, the value that has the attractive property of being consistent with Kolmogorov's scaling for lower wavenumbers. On the other hand, both DIA (Kraichnan 1959) and the quasi-normal theories (Orszag 1973) predict $\alpha=3$. The recent numerical studies by Kida $e t$ al. (1992) with data for $k / k_{d}<4$ found that $\alpha<0$. Kida et al. argue that the negative value for $\alpha$ is connected to the fact that in the dissipation range, the energy transfer is dominated by nonlocal triads. See also the study by Zhou (1994). Domaradzki (1992) obtains $\alpha=-2$, deduced from a scaling ansatz for energy transfer. On the other hand, Chen et al. (1993) have confirmed the prediction of the DIA and the EDQNM for $4<k / k_{d}<10$, obtaining $\alpha=3.3$ and $\beta=7.1$. Since the Reynolds numbers have been so low, some concerns still remain. Using very large wind tunnel experiments, recently, Saddoughi \& Veeravalli (1994) found $\beta=5.2$, for $0.2<k / k_{d}<1$, at $R_{\lambda} \approx 600$, in accordance with other works (see e.g. Sanada 1992, Kida et al. 1992, Kida \& Murakami 1990). 


\section{Analysis}

In this note, we concentrate on the determination of $\alpha$ and $\beta$, and their possible dependence on the Reynolds number and wavelength, from numerical simulations. A series of direct numerical simulations of incompressible Navier-Stokes equations has been carried out in a periodic box with sides $2 \pi$. We use a pseudo-spectral code on the CM-5 parallel supercomputer at Los Alamos National Laboratory. The resolution ranges from $256^{3}$ (maximum wavenumber $k_{\max }=128$ ), to $512^{3}\left(k_{\max }=256\right)$. The system is initialized with a random velocity field with Gaussian statistics. These Fourier velocity amplitudes give an energy spectrum of $k^{4} \exp \left[-\left(k / k_{0}\right)^{2}\right]$, with $k_{0} \sim 5$. If the system is then left to evolve, nonlinear interactions will redistribute the energy among all available modes. This familiar process delivers energy (in a statistical sense) from larger scales to smaller scales, until the excited wavenumbers are high enough for the viscosity to efficiently dissipate energy. In order to achieve a steady state, energy is pumped into the system by stirring it at the large scales. Specifically, the mode amplitudes for $k=1$ and 2 (the largest scales) are forced to values consistent with Kolmogorov's $-5 / 3$ law for the energy spectrum. Once statistically steady values of $R_{\lambda}$ and dissipation per unit mass, $\epsilon$, were reached, several snapshots of the energy spectrum, spanning several eddy-turnover characteristic times, were averaged and analyzed.

In Table I, the simulations are sorted by increasing $R_{\lambda}=v_{0} \lambda / \nu$, the Taylor microscale Reynolds number, where $v_{0}$ is the mean square velocity, and $\lambda$ is the Taylor microscale. The table also displays information about how well the dissipation range is resolved (column under " $k_{\max } / k_{d}$ "). Notice that although for some of the simulations $k_{\max }$ is of the order of $k_{d}$, for six of the simulations $k / k_{d} \sim 5$, i.e., fairly deep into the dissipation range. In particular, the third entry in the Table $\left(R_{\lambda}=38\right)$ corresponds to a double-precision, $512^{3}$ DNS, which constitutes to the best knowledge of the authors the memory-wise most demanding turbulence simulation to date.

To investigate the possible values for $\alpha$ and $\beta$, the quantity, $d \ln E(k) / d \ln k$, as a function of $k / k_{d}$, was computed from the energy spectrum, for each of the runs. If the functional form suggested in (1) is correct, then $d \ln E(k) / d \ln k$ should be close to a straight line. The slope, $\beta / k_{d}$, and the intersection of the line with the vertical axis (at $d \ln E(k) / d \ln k=\alpha$ ) were then determined 
by means of a least-squares fit to restricted ranges of $k / k_{d}$.

Figure 1 shows $d \ln E(k) / d \ln k$ vs $k / k_{d}$ for the run that best resolves the dissipation range (run 3 in Table I). Two different slopes are found for the lower and higher wavenumber regions, the latter one being steeper. The transition occurs at $k / k_{d} \sim 4$. These are common features for the simulations that penetrate deep into the dissipation range, namely, the two-slope behavior for $d \ln E(k) / d \ln k$ vs. $k / k_{d}$, and the transition between the two slopes at $k / k_{d} \sim 4$. It is possible that this shift in slope indicates a change in the nature of the balance of the triadic interactions. We have calculated the transfer energy function $T(k \mid p, q)$ that measures the amount of energy transferred to wavenumber $k$, due to interactions with wavenumbers $p$ and $q$. A preliminary study of $T(k \mid p, q)$ has not proven to be very revealing.

A bump at $k / k_{d} \sim 4$ in Fig. 1 accompanies the occurrence of a change in the energy slope. Similar bumps at much smaller $k / k_{d}$ have been ascribed to a "bottleneck" effect (Lohse \& MüllerGroeling 1995) in which triadic interactions that can cascade energy out of the region are inhibited because the spectrum falls off rapidly as $k / k_{d}$ increases. The energy pileup in our study seems to be of a weaker nature than the one reported by Lohse and Müller-Groeling. In that work, the secondorder derivative of the energy with respect to $k$, is non-monotonic. In our study, the third derivative is the non-monotonic one. A completely satisfying explanation of this effect is more difficult since there is not a clearly conserved quantity associated with the dissipation range, whereas, in the inertial range, the energy flux is independent of the wavenumber, i.e., it is conserved. It could be conjectured through these observations that the sharp change in the local slope of the energy spectrum, will result in an energy pileup close to the transition point, due to the change of the nature of the balance of the triadic interactions.

From the energy spectrum shown in Fig. 1 we computed $\alpha$ and $\beta$ as a function of $k / k_{d}$. The result is displayed in Figure 2. Panels a) and b) show $\alpha$ and $\beta$, respectively, as a function of $k / k_{d}$ for run 3 in Table I. These values were obtained as the local $\alpha$ and $\beta$ (i.e., local intersection with the $y$-axis and local slope) of $d \ln E / d \ln k$. The values for $\alpha$ and $\beta$ assigned to a particular wavenumber, were computed as a least-squares fit to the energy spectrum in the range $\left[k / k_{d}-0.9, k / k_{d}+0.9\right]$. For $\alpha$ we observe a tendency to be negative for lower $k$ and positive for higher wavenumbers. The 
plot in panel b) shows that $\beta$ is fairly constant at a value between five and six, except at $k / k_{d} \sim 4$, where it drops to about four. For both parameters we observe that a plateau is reached for $k / k_{d}<2$ and $k / k_{d}>8$. We note that this plateau is not expected to extend to the inertial range scales since that would imply that the energy spectrum would have the same functional form in the dissipation and in the inertial ranges.

Figure 3 shows the dependence of the coefficients $\alpha$ and $\beta$ on $R_{\lambda}$. Panel a) displays $\alpha$ vs $R_{\lambda}$. Points indicated by the " $x$ " symbol, correspond to measurements done for $k / k_{d}<3$ for all runs in Table I; the diamonds correspond to $k / k_{d}>6$ for runs 1 through 4 . For $\alpha$, all values obtained from the range $k / k_{d}<3$ are consistently negative, within the range -1 to -2 , in the neighbourhood of the Kolmogorov's value $-5 / 3$. The four points obtained in the more dissipative range, are positive, in the range $3-6$. This two-value behavior of $\alpha$ is a preliminary result. It might be possible that the region of positive values for $\alpha$ can be extended beyond $R_{\lambda} \sim 40-50$ if these values are evaluated from computations that better resolve the dissipation range. In other words, it is possible that the negative values for $\alpha$ appear because they are being computed from the near-dissipation range.

For $\beta$, the values measured in the range $k / k_{d}>6$ (diamonds), are consistently higher than those measured in the range $k / k_{d}<3$. Recently, Sirovich et al. derived the energy spectrum (for both inertial range and dissipation range) from the third order structure function (Sirovich. Smith \& Yakhot 1994). The value of $\beta$ obtained in that work is a function of the skewness and the dissipation length. The skewness is believed to be independent of $R_{\lambda}$ (Kerr 1985), and thus it is likely that the behavior of $\beta$ observed in Fig. $2 \mathrm{~b}$ ) is consistent with the derivation in Sirovich et al. (1994).

\section{Conclusion}

These values for $\alpha$ and $\beta$ are consistent with those reported by Chen et al. (1993) obtained with a DNS with a dissipation range resolution comparable to run number three in Table I, but with a lower Reynolds number. The results presented here, and particularly in reference to Fig. 3, might indicate that the exponential functional form (1) set forth for the energy spectrum in the dissipation range, may not be the most appropriate guess for the near-dissipation range, i.e., 
$k / k_{d}<4$. Solidly establishing these parameters as a function of $R_{\lambda}$ will require next-generation computer capabilities. At this point we are faced with the dilemma of having to choose between performing DNS with relatively high values for $R_{\lambda}$ or having a relatively well-resolved dissipation range $\left(k_{\text {max }} / k_{d}>>1\right)$ at a relatively low Reynolds number. This study shows a definite trend, and it should be taken as an effort to shed some light on these issues by using state-of-the-art DNS.

Computations were performed on the CM5 at the Advanced Computing Laboratory at Los Alamos, and on the SP-1 and SP-2 at the IBM Watson Research Center. Discussions with N. Cao, D. Lohse, Z.-S. She, and V. Yakhot are gratefully acknowledged. 


\section{REFERENCES}

[1] CHEN S., DOOLEN G.D., HERRING J.R., KRAICHNAN R.H., ORSZAG S.A., \& SHE Z.S. 1993 Phys. Rev. Lett. 70, 3051.

[2] DOMARADZKI J. A. 1992 Phys. Fluids A, 4, 2037.

[3] FOIAS C., MANLEY O., \& SIROVICH L. 1990 Phys. Fluids A 2, 464.

[4] KIDA S., KRAICHNAN R.H., ROGALLO R.S., WALEFFE F., \& ZHOU Y. 1992 in Proceedings of the Summer Program 1992, Center for Turbulence Research, Stanford University and NASA/AMES Research Center.

[5] KIDA S. \& MURAKAMI Y. 1987 Phys. Fluids A, 30, 2030; KERR R. 1990 J. Fluid Mech. 211, 309 .

[6] KERR R. 1985 J. Fluid Mech. 153, 31.

[7] KOLMOGOROV A.N. 1941 C. R. Acad. Sci. URSS 30301.

[8] KRAICHNAN R.H. 1959 J. Fluid Mech. 5, 497.

[9] LOHSE D. \& A. MÜLlER-GRoEling A. 1995 Phys. Rev. Lett. 74, 1747.

[10] MANLEY O.P. 1992 Phys. Fluids A 4, 1320.

[11] MCCOMB W.D. 1990 Chapter 2, Clarendon, Oxford.

[12] NOVIKOV E.A. 1961 Dokl. Akad. Nauk. SSR 139, 331.

[13] ORSZAG S.A. 1973 in Fluid Dynamics 1973, Les Houches Summer School of Theoretical Physics, Gordon and Breachi, 237.

[14] SADDOUGHI S.G. \& VEERAVALLI S.V. 1994 J. Fluid Mech. 268, 333.

[15] SANADA T. 1992 Phys. Fluids A 4, 1086.

[16] SIROVICH L., SMITH L., \& YAKHOT V. 1994 Phys. Rev. Lett. 72, 344. 
[17] SMITH L.M. \& REYNOLDS W.C. 1991 Phys. Fluids A 3, 992.

[18] SREENIVASAN K.R. 1985 J. Fluid Mech. 151, 81.

[19] TOWNSEND A.A. 1951 Proc. R. Soc. London Ser. A 208, 534.

[20] ZHOU Y. 1993 Phys. Fluids A, 5, 2511. 


\section{TABLES}

\begin{tabular}{|c|c|c|c|c|}
\hline \hline Run & $\mathrm{R}_{\lambda}$ & $\mathrm{k}_{d}$ & $\mathrm{k}_{\max } / k_{d}$ & Resolution \\
\hline 1 & 20.9 & 9.7 & 10 & 256 \\
2 & 33.0 & 13.9 & 8 & 256 \\
3 & 38.0 & 16.6 & 12 & 512 \\
4 & 39.1 & 16.7 & 7 & 256 \\
5 & 48.4 & 19.9 & 5.8 & 256 \\
6 & 52.9 & 23.6 & 4.7 & 256 \\
7 & 67.9 & 33.4 & 3.3 & 256 \\
8 & 101.9 & 55.7 & 4.4 & 512 \\
\hline \hline
\end{tabular}

TABLE I. Direct numerical simulations on a $2 \pi^{3}$ periodic domain. Resolution ranges are $128^{3}$, $256^{3}$, and $512^{3}$. The third entry corresponds to a $512^{3}$ resolution, double precision run. 


\section{FIGURES}

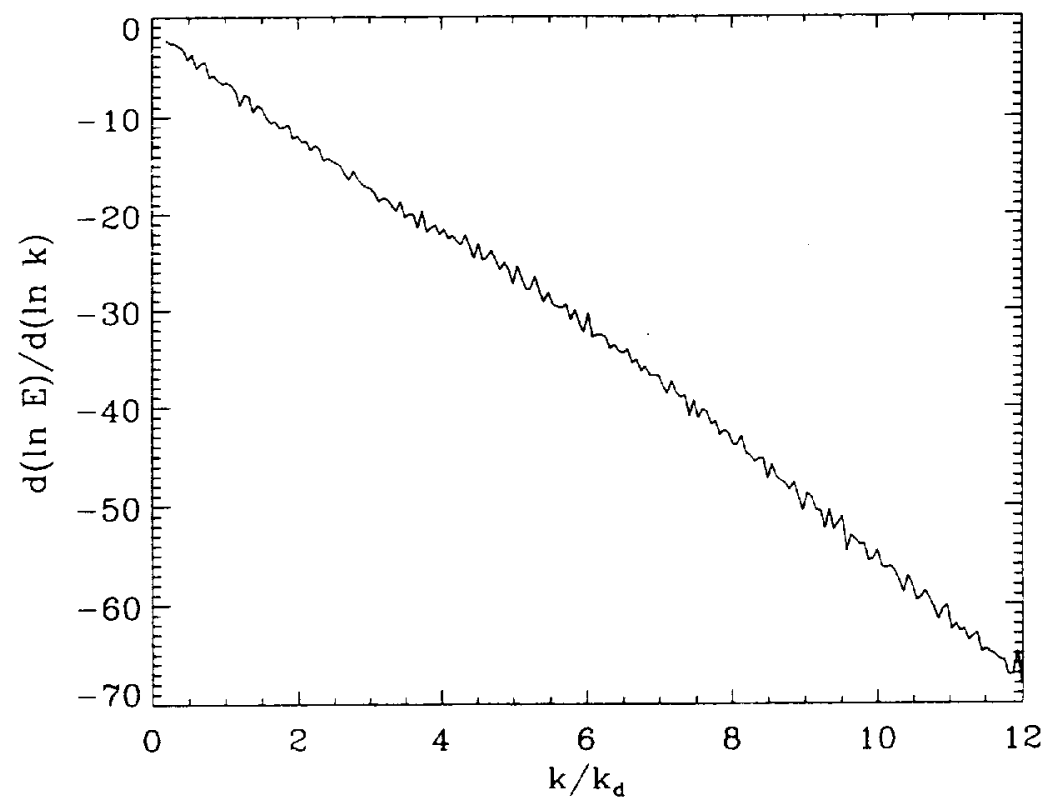

FIG. 1. $d \ln E / d \ln k$ vs. $k / k_{d}$ for run 3 in Table I. Two slopes are observed in the low and high wavenumber range. The energy spectrum $E(k)$ is computed by averaging the energy spectrum over several eddy-turnover times after the system has reached a steady state. 



FIG. 2. Local values of $\alpha$ and $\beta$ as a function of $k / k_{d}$, calculated for run 3 in Table I. 

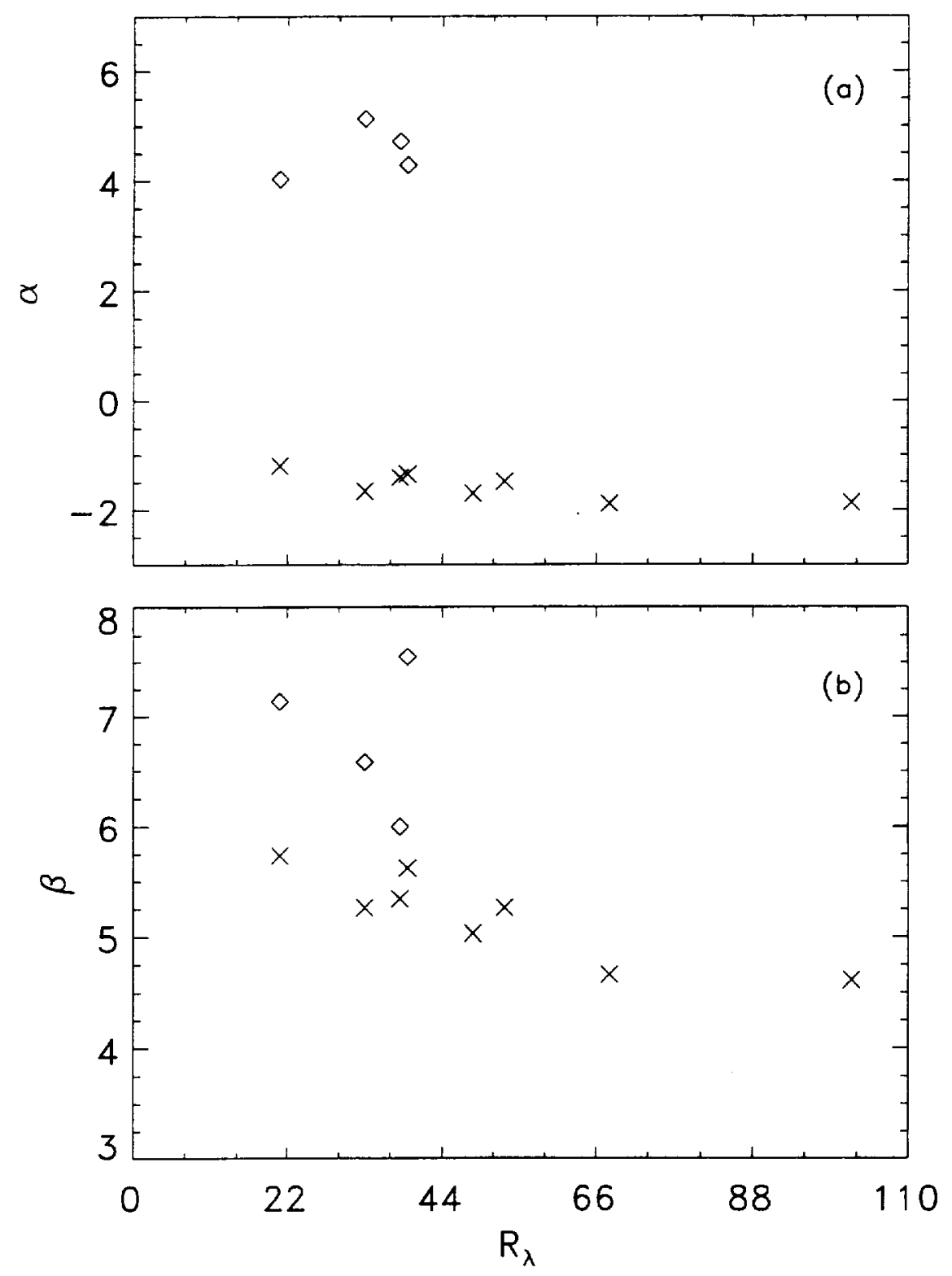

FIG. 3. Parameters $\alpha$ a), and $\beta$ b), as a function of $R_{\lambda}$. These values are calculated by performing a least-squares fit to $d \ln E / d \ln k$ vs. $k / k_{d}$ (see Fig. 1). Points indicated by the " $\times$ " symbol, correspond to measurements done in the range $k / k_{d}<3$ for all runs in Table I; the diamonds correspond to $k / k_{d}>6$ for runs 1 through 4 . 



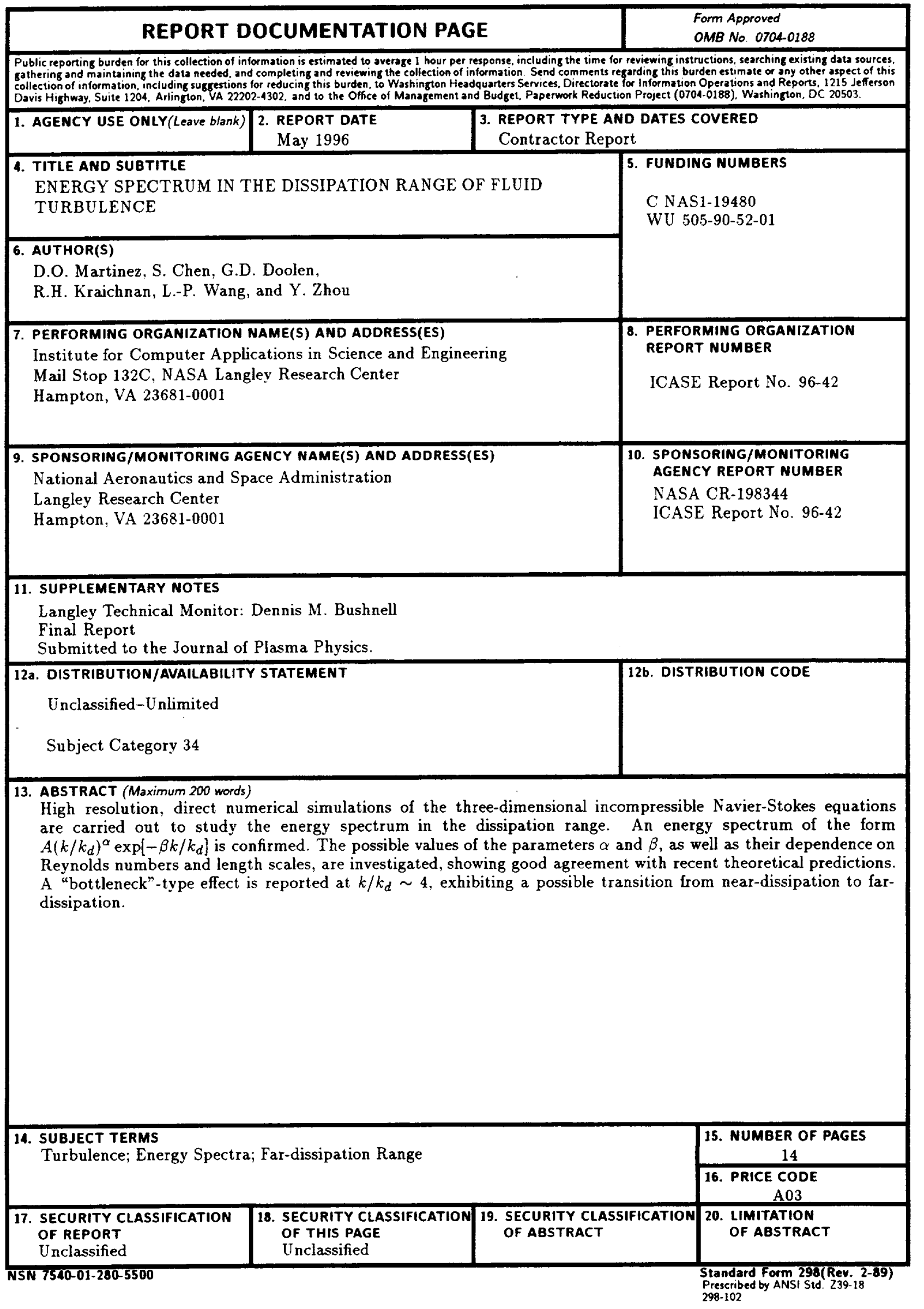

